\title{
Longitudinal Association of Handgrip Strength with Medical Use and the Risk of Hospitalization in Korean Adults
}

\author{
Junghoon $\mathrm{Kim}^{1 *}$ \\ ${ }^{1}$ Sports and Exercise Medicine Laboratory, Korea Maritime \& Ocean University, Busan, Republic of Korea
}

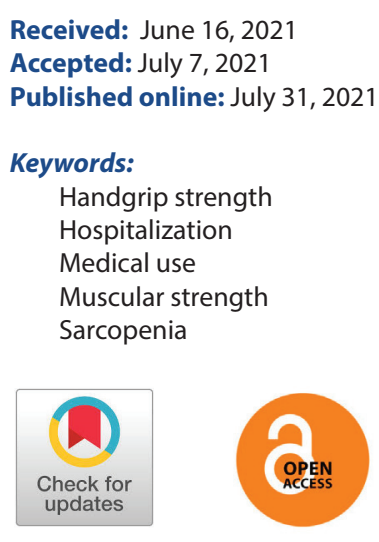

\section{ABSTRACT}

OBJECTIVES Age-related loss of muscular strength may contribute to medical health condition. Decreased handgrip strength is associated with an increased risk of chronic diseases, disability, and mortality. However, it is not known whether handgrip strength is also linked with hospitalization and medical use from the long-term cohort study in middle-aged adults. Thus, we aimed to investigate the association of handgrip strength levels, medical use, and hospitalization in Korean adults.

METHODS This large prospective study was based on data from Korean Longitudinal Study of Ageing 2006 to 2018 . In total, 9,228 participants aged $\geq 45$ years were included for this study. Handgrip strength was measured using dynamometer, and classified into quartiles by sex-specific cutoff point.

RESULTS Significantly higher hazard ratio (HR) for hospitalization was observed in lower handgrip strength (HR: $1.22,95 \% \mathrm{Cl}: 1.11-1.34)$ compared to higher handgrip strength (reference) in the fully adjusted model. We also found longitudinal association of handgrip strength levels and the use of hospitalization and outpatient medical care during 12 years following period using mixed effect modes with time-dependent interaction.

CONCLUSIONS Our results suggests that lower handgrip strength was associated with increased the risk of hospitalization and use of medical care in Korean population. This study highlights the maintaining of muscular strength may play an important role in the reduction of risk for hospitalization and medical use by preventing chronic diseases.

(c) The Asian Society of Kinesiology and the Korean Academy of Kinesiology

\section{Introduction}

Loss of muscular strength and skeletal muscle mass has been considered as typical physical changes with ageing [1]. The skeletal muscle declines by about $0.8 \%$ per year after 40 years of age, and it has been reported that the skeletal muscle mass declines by about $15 \%$ in 10 years after 70 years of age [2]. In this way, a condition characterized by age-related loss of skeletal muscle mass and muscular strength is referred to

*Correspondence: Junghoon Kim, Ph.D., Sports and Exercise Medicine Laboratory, Korea Maritime \& Ocean University, Busan, Republic of Korea; TEL: +82-51-410-4792; E-mail: junghoonkim@kmou.ac.kr as sarcopenia $[3,4]$.

Sarcopenia is a chronic health condition that can cause chronic diseases, disability, and which increases dependence, and risk of all-cause mortality [5-7]. Recent studies have been reported that sarcopenia contribute to the increased risk of physical disabilities such as decreased gait speed and falls in elderly population [8]. In addition, other experimental and epidemiological studies mentioned that sarcopenia is one of risk factors that causes concomitant diseases such as geriatric chronic diseases and metabolic disorders $[9,10]$. Therefore, prevention of sarcopenia may be an effective strategy in 
improving the quality of life, physical independence, and preventing chronic diseases.

Several studies have been attempted the evaluation and diagnosis of sarcopenia. Recent studies and working groups have suggested diagnosing sarcopenia using the handgrip strength as a decreased muscular function. In fact, several previous studies investigated that handgrip strength has a prospective association with the increased risk of chronic diseases, cognitive function and mortality [11-13]. Most of previous studies have investigated relationship between decrease in muscle mass and chronic health conditions of individuals. Moreover, there are only a few studies investigating the relationship between muscular function and medical use including the risk of hospitalization [14]. Especially, the association of handgrip strength and health condition such as medical use and the risk of hospitalization has not been explored in general Korean population. Therefore, in our study, we aimed to investigate the prospective associations of decreased handgrip strength and medical use and the risk of hospitalization in the general Korean adults aged $\geq 45$ years in baseline.

\section{Materials and Methods}

\section{Participants}

The present study used the data from the Korean Longitudinal Study of Ageing (KLoSA). The KLoSA is an ongoing national prospective cohort study of representative Korean adults aged $\geq 45$ years. Additional details about the study protocol including participants, design and methods used in this study has been reported in previous study. This study was restricted to participants aged $\geq 45$ years and who completed the health-related behaviors and medical health conditions $(n=10,254)$. We excluded 818 participants with missing data of handgrip strength, 200 participants with missing data on body mass index (BMI), and 8 participants with missing data on other confounding factors. In total, 9,228 participants were included for this study. All participants provided written informed consent, and the KLoSA study was approved by the Institutional Review Board of the Korea Employment Information Service.

\section{Measurement of handgrip strength}

Handgrip strength was assessed using the dynamometer from each participant. The maximum handgrip strength was measured three times with the standing position. We calculated the mean of the average value from maximum value of left and right hands $[15,16]$. To examine the association of different levels of handgrip strength on the medical use, we divided participants into quartiles of handgrip strength by sex $(<28.5,28.5-32.7,32.8-37.2$, and $\geq 37.3 \mathrm{~kg}$ for male, $<17.0$, $17.0-19.9,20.0-22.9$, and $\geq 23.0 \mathrm{~kg}$ for female).

\section{Medical use and hospitalization}

In this study, main outcomes were the use of outpatient and hospitalization medical care (days/years). All medical information was examined every 2 years from baseline (2006) to the end of follow up at 2018. All of the information for days for the use of medical care obtained from the medical receipt. To analyze the risk of hospitalization, we also generated variable for hospitalization defined as those who were hospitalized at least once a year.

\section{Other covariates}

We also used baseline characteristics from the following variables that have been shown to be associated with the risk of hospitalization and the use of medical care as potential confounding factors: demographics (age, sex, household income and education level), lifestyle factors (smoking status, alcohol consumption, and physical activity levels), self-reported health status, and clinical health conditions (hypertension, diabetes, CVD, stroke, cancer, and osteoarthritis). The moderate-to-vigorous physical activity (MVPA) was measured using the questionnaire International Physical Activity Questionnaire [17], after which participants were divided into two groups based on the guidelines of the World Health Organization (WHO) $[18,19]$ recommended levels for physical activity ( $<150 \mathrm{~min} /$ week or $\geq 150 \mathrm{~min} /$ week). Educational level was classified as " $\leq$ middle school", "high school" or " $\geq$ college". Smoking status of participants were divided into three groups as "never", "former" or "current" and alcohol consumption as "never", " $\leq$ once/week", "2-3 times/ week”, or “ $\geq 4$ times/week”. 


\section{Data analysis}

All data were analyzed using SAS survey procedures (ver. 9.4, SAS Institute, Cary, NC). Participant baseline characteristics were described as means \pm standard deviation (SD) for continuous variables and percentages (\%) for categorical. All handgrip strength were classified according to sex-specific quartiles based on the baseline handgrip strength levels and the highest quartile group used as the reference group in all analysis. The statistical significance of the handgrip strength levels were evaluated using the regression models for continuous variables and chi-square tests for categorical variables. To examine the prospective effect of handgrip strength on outpatient use and hospitalization days, we used mixed effect models for repeated measured analysis. Furthermore, cox proportional hazard models were used to predict the risk of hospitalization from handgrip strength levels. To assess the influence of potential confounding factors, four models were sequentially developed. Model 1 was unadjusted; model 2 was adjusted for sex, age, household income, and education level; model 3 was additionally adjusted for BMI, smoking status, alcohol consumption, and physical activity level; model 4 additionally adjusted for chronic clinical health status at baseline (hypertension, diabetes, cardiovascular disease (CVD), and osteoarthritis $(\mathrm{OA})$ ). Statistical significance was considered as $P$-value $<0.05$.

Table 1. Participants characteristics according to handgrip strength levels

\begin{tabular}{|c|c|c|c|c|c|c|c|c|c|c|c|}
\hline & \multicolumn{10}{|c|}{ Handgrip strength levels } & \multirow{2}{*}{ P-value } \\
\hline & \multicolumn{2}{|c|}{ Q1 (n=2,273) } & \multicolumn{2}{|c|}{ Q2 $(n=2,233)$} & \multicolumn{2}{|c|}{ Q3 (n=2,322) } & \multicolumn{2}{|c|}{ Q4 $(n=2,400)$} & \multicolumn{2}{|c|}{ Overall $(n=9,228)$} & \\
\hline Male & 1,007 & $(44.3)$ & 1,064 & $(47.7)$ & 1,028 & $(44.3)$ & 1,032 & $(43.0)$ & 4,131 & $(44.8)$ & 0.0119 \\
\hline Female & 1,266 & $(55.7)$ & 1,169 & $(52.4)$ & 1,294 & $(55.7)$ & 1,368 & $(57.0)$ & 5,097 & $(55.2)$ & \\
\hline \multicolumn{12}{|l|}{ Age } \\
\hline$<65$ years & 665 & $(29.3)$ & 1,220 & $(54.6)$ & 1,764 & $(76.0)$ & 2,154 & $(89.8)$ & 5,803 & $(62.9)$ & $<.0001$ \\
\hline$\geq 65$ years & 1,608 & $(70.7)$ & 1,013 & $(45.4)$ & 558 & $(24.0)$ & 246 & $(10.3)$ & 3,425 & $(37.1)$ & \\
\hline \multicolumn{12}{|c|}{ Household income [n (\%)] } \\
\hline Q1 & 814 & $(35.8)$ & 594 & $(26.6)$ & 514 & $(22.1)$ & 409 & $(17.0)$ & 2,331 & $(25.3)$ & $<.0001$ \\
\hline Q2 & 799 & $(35.2)$ & 723 & $(32.4)$ & 593 & $(25.5)$ & 550 & $(22.9)$ & 2,665 & $(28.9)$ & \\
\hline Q3 & 336 & $(14.8)$ & 459 & $(20.6)$ & 557 & $(24.0)$ & 583 & $(24.3)$ & 1,935 & $(21.0)$ & \\
\hline Q4 & 324 & (14.3) & 457 & $(20.5)$ & 658 & $(28.3)$ & 858 & $(35.8)$ & 2,297 & $(24.9)$ & \\
\hline \multicolumn{12}{|l|}{ Education level } \\
\hline$\leq$ Middle school & 1,543 & $(67.9)$ & 1,099 & $(49.2)$ & 820 & $(35.3)$ & 585 & $(24.4)$ & 4,047 & $(43.9)$ & $<.0001$ \\
\hline High school & 285 & $(12.5)$ & 381 & $(17.1)$ & 454 & (19.6) & 448 & $(18.7)$ & 1,568 & $(17.0)$ & \\
\hline$\geq$ College & 445 & (19.6) & 753 & $(33.7)$ & 1,048 & $(45.1)$ & 1,367 & $(57.0)$ & 3,613 & $(39.2)$ & \\
\hline \multicolumn{12}{|l|}{ Smoking status } \\
\hline Never smoker & 1,592 & $(70.0)$ & 1,531 & $(68.6)$ & 1,659 & (71.5) & 1,735 & $(72.3)$ & 6,517 & $(70.6)$ & 0.0002 \\
\hline Former smoker & 256 & $(11.3)$ & 240 & $(10.8)$ & 194 & $(8.4)$ & 188 & $(7.8)$ & 878 & $(9.5)$ & \\
\hline Current smoker & 425 & (18.7) & 462 & $(20.7)$ & 469 & $(20.2)$ & 477 & (19.9) & 1,833 & (19.9) & \\
\hline \multicolumn{12}{|c|}{ Alcohol consumption } \\
\hline None & 691 & $(30.4)$ & 884 & (39.6) & 988 & $(42.6)$ & 1,098 & $(45.8)$ & 3,661 & (39.7) & $<.0001$ \\
\hline Once a week & 240 & $(10.6)$ & 157 & $(7.0)$ & 105 & $(4.5)$ & 71 & $(3.0)$ & 573 & $(6.2)$ & \\
\hline$\geq 2$ times/week & 1,342 & $(59.0)$ & 1,192 & $(53.4)$ & 1,229 & $(52.9)$ & 1,231 & $(51.3)$ & 4,994 & $(54.1)$ & \\
\hline \multicolumn{12}{|l|}{ Physical activity } \\
\hline$<150 \mathrm{~min} /$ week & 1,794 & (78.9) & 1,581 & $(70.8)$ & 1,578 & $(68.0)$ & 1,601 & $(66.7)$ & 6,554 & $(71.0)$ & $<.0001$ \\
\hline$\geq 150 \mathrm{~min} /$ week & 479 & $(21.1)$ & 652 & $(29.2)$ & 744 & $(32.0)$ & 799 & $(33.3)$ & 2,674 & $(29.0)$ & \\
\hline
\end{tabular}




\section{Results}

The participant's general characteristics according to handgrip strength was presented in $<$ Table $1>$. Overall, 44.8\% $(n=4,131)$ participants were male and $37.1 \%(n=3,425)$ participants was older population, $19.9 \%(n=1,833)$ participants were current smoker, and $29.0 \%(n=2,674)$ participants were physically active population. As shown $<$ Table 1>, the frequency of older population, household income, education level, smoking status, alcohol consumption, and physical activity level were significantly different across handgrip strength levels (all P-value $<0.05$ ).

$<$ Table $2>$ shown that the participant's health status in baseline by handgrip strength. The frequency of obese, and self-reported and clinical health status (hypertension, diabetes, CVD, cancer, liver disease, and osteoarthritis) were significantly different across handgrip strength (all $P$-value $<0.05)$.

Cox-proportional hazard models were used to evaluate the risk for hospitalization between groups according to handgrip strength levels $<$ Table $3>$. Significantly higher HR for hospitalization was observed in lower handgrip strength (HR: 1.36, 95\% CI:1.24-1.84) compared to higher handgrip strength (reference) in the unadjusted model (Model 1). Moreover, adjusted model for age, sex, household income, and education level, the levels of handgrip strength was associated with the risk of hospitalization (Model 2). We also found significant association of handgrip strength and risk of hospitalization in the additionally adjusted model for body mass index, smoking status, alcohol consumption, and physical activity level (Model $3)$. When further adjusted self-reported and clinical health status at baseline, the significant trends were maintained (Model 4).

$<$ Figure $1>$ and $<$ Figure $2>$ shown that the longitudinal association of handgrip strength levels and the use of hospitalization and outpatient medical care using mixed effect modes after full adjusted for covariates. Compared with highest level of handgrip strength, the days of outpatient care was significantly higher in lower handgrip strength group $<$ Figure 1 $>$. Moreover, compared with highest level of handgrip strength, the days of hospitalization was significantly higher in lower handgrip strength group in $<$ Figure 2 $>$.

Table 2. Clinical health conditions according to handgrip strength levels

\begin{tabular}{|c|c|c|c|c|c|c|c|c|c|c|c|}
\hline & \multicolumn{10}{|c|}{ Handgrip strength levels } & \multirow{2}{*}{ P-value } \\
\hline & \multicolumn{2}{|c|}{ Q1 (n=2,273) } & \multicolumn{2}{|c|}{ Q2 (n=2,233) } & \multicolumn{2}{|c|}{ Q3 $(n=2,322)$} & \multicolumn{2}{|c|}{ Q4 $(n=2,400)$} & \multicolumn{2}{|c|}{ Overall $(n=9,228)$} & \\
\hline Non-obese & 1,855 & $(81.6)$ & 1,756 & $(78.6)$ & 1,767 & $(76.1)$ & 1,708 & $(71.2)$ & 7,086 & $(76.8)$ & $<.0001$ \\
\hline Obese & 418 & $(18.4)$ & 477 & $(21.4)$ & 555 & $(23.9)$ & 692 & $(28.8)$ & 2,142 & $(23.2)$ & \\
\hline \multicolumn{12}{|c|}{ Self-reported health status } \\
\hline Excellent & 34 & $(1.5)$ & 68 & $(3.1)$ & 103 & $(4.4)$ & 138 & $(5.8)$ & 343 & $(3.7)$ & $<.0001$ \\
\hline Very Good & 388 & $(17.1)$ & 684 & $(30.6)$ & 1,019 & $(43.9)$ & 1,281 & $(53.4)$ & 3,372 & $(36.5)$ & \\
\hline Good & 739 & $(32.5)$ & 831 & $(37.2)$ & 753 & $(32.4)$ & 700 & $(29.2)$ & 3,023 & $(32.8)$ & \\
\hline Fair & 848 & $(37.3)$ & 542 & $(24.3)$ & 389 & $(16.8)$ & 266 & $(11.1)$ & 2,045 & $(22.2)$ & \\
\hline Poor & 264 & $(11.6)$ & 108 & $(4.8)$ & 58 & $(2.5)$ & 15 & $(0.6)$ & 445 & $(4.8)$ & \\
\hline \multicolumn{12}{|c|}{ Clinical health status } \\
\hline Hypertension & 834 & $(36.7)$ & 653 & $(29.2)$ & 556 & $(23.9)$ & 425 & (17.7) & 2,468 & $(26.7)$ & $<.0001$ \\
\hline Diabetes & 406 & $(17.9)$ & 295 & $(13.2)$ & 219 & $(9.4)$ & 155 & $(6.5)$ & 1,075 & $(11.7)$ & $<.0001$ \\
\hline CVD & 182 & $(8.0)$ & 118 & $(5.3)$ & 68 & $(2.9)$ & 52 & $(2.2)$ & 420 & (4.6) & $<.0001$ \\
\hline Stroke & 110 & $(4.8)$ & 62 & $(2.8)$ & 40 & $(1.7)$ & 19 & $(0.8)$ & 231 & $(2.5)$ & $<.0001$ \\
\hline Cancer & 72 & $(3.2)$ & 49 & $(2.2)$ & 46 & $(2.0)$ & 34 & (1.4) & 201 & $(2.2)$ & $<.0001$ \\
\hline Liver disease & 42 & (1.9) & 45 & $(2.0)$ & 32 & $(1.4)$ & 29 & $(1.2)$ & 148 & (1.6) & 0.0945 \\
\hline Osteoarthrosis & 570 & $(25.1)$ & 352 & $(15.8)$ & 306 & $(13.2)$ & 203 & (8.5) & 1,431 & $(15.5)$ & $<.0001$ \\
\hline
\end{tabular}


Table 3. HR ( $95 \% \mathrm{Cls})$ for hospitalization by category of handgrip strength

\begin{tabular}{|c|c|c|c|c|c|c|c|c|}
\hline \multirow{2}{*}{ Handgrip strength } & \multicolumn{2}{|c|}{ Model 1} & \multicolumn{2}{|c|}{ Model 2} & \multicolumn{2}{|c|}{ Model 3} & \multicolumn{2}{|r|}{ Model 4} \\
\hline & HR & $(95 \% \mathrm{CI})$ & HR & $(95 \% \mathrm{CI})$ & HR & $(95 \% \mathrm{Cl})$ & HR & $(95 \% \mathrm{CI})$ \\
\hline Q1 $(n=1,894)$ & 1.36 & $(1.24-1.48)^{* * *}$ & 1.23 & $(1.12-1.34)^{* * *}$ & 1.26 & $(1.15-1.38)^{* * *}$ & 1.22 & $(1.11-1.34)^{* * *}$ \\
\hline Q2 $(n=1,855)$ & 1.15 & $(1.05-1.27)^{* * *}$ & 1.06 & $(0.96-1.16)$ & 1.06 & $(0.96-1.17)$ & 1.05 & $(0.95-1.16)$ \\
\hline Q3 $(n=1,908)$ & 1.10 & $(0.99-1.21)$ & 1.02 & $(0.92-1.12)$ & 1.04 & $(0.94-1.14)$ & 1.02 & $(0.92-1.12)$ \\
\hline $\mathrm{Q} 4(\mathrm{n}=1,945)$ & 1.00 & (reference) & 1.00 & (reference) & 1.00 & (reference) & 1.00 & (reference) \\
\hline P-for trend & & 0.003 & & .015 & & 0.023 & & 0.023 \\
\hline
\end{tabular}

Model 1 unadjusted model; Model 2 adjusted for age, sex, education level, and household income; Model 3 adjusted for Model 2 covariates plus body mass index, smoking status, alcohol consumption, and physical activity level; and Model 4 adjusted for Model 3 covariates plus chronic clinical health status at baseline (hypertension, diabetes, CVD, and OA). ${ }^{* *} P$-value $<0.001$ vs. reference group.

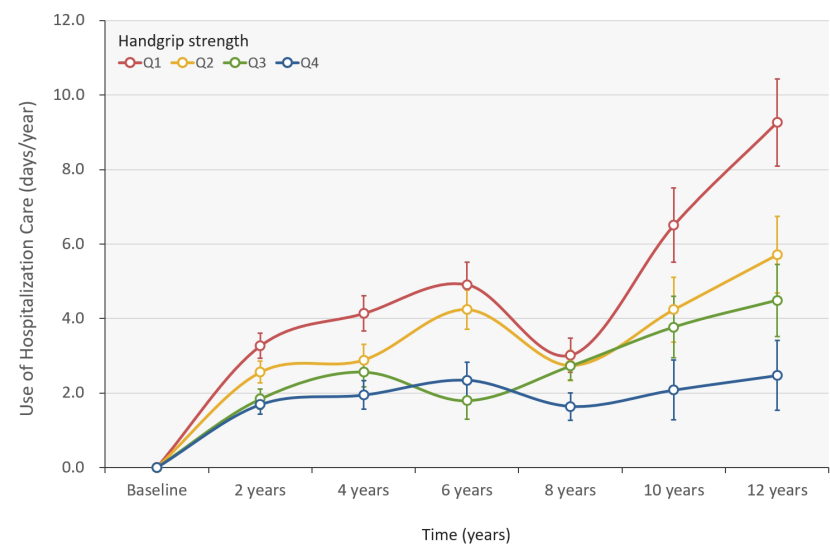

Figure 1. The hospitalization (days/years) according to handgrip strength levels.

Values were mean days (SE). Fully adjusted model included age, sex, educational level, household income, smoking status, alcohol consumption, MVPA, and clinical health status at baseline as a covariate. MVPA, moderateto-vigorous physical activity.

We also evaluated the time-dependent interaction according to handgrip strength level on use of hospitalization and outpatient medical care. The significant interaction observed by handgrip strength on the change of use of hospitalization and outpatient care during 12 years following period (group $\times$ time, all $P$ for interaction $<0.001,<$ Figure $1>$ and $<$ Figure $2>$ ).

\section{Discussion}

Our results suggest that decreased handgrip strength levels contribute independently to increased risk of hospitalization during 12 years follow up in general Korean population. In addition, we found that the increased use of medical care for

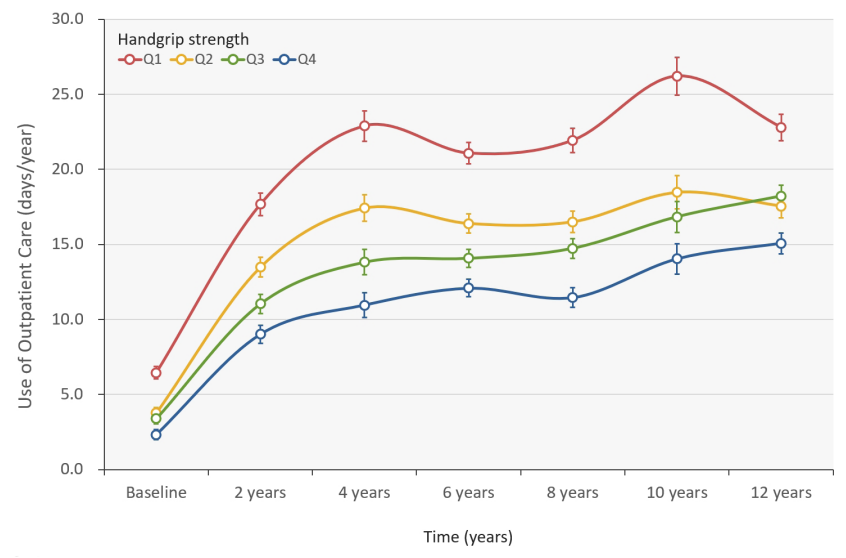

Figure 2. The use of outpatient care (days/years) according to handgrip strength levels.

Values were mean days (SE). Fully adjusted model included age, sex, educational level, household income, smoking status, alcohol consumption, MVPA, and clinical health status at baseline as a covariate. MVPA, moderateto-vigorous physical activity.

outpatient and hospitalization in the lower handgrip strength group than in either of the individual conditions. The risk of hospitalization in lower handgrip strength was 1.22 times higher compared with the higher handgrip strength group. The estimated days of outpatient medical use in lower handgrip strength group (mean, 9.26 days/years, 95\% CI, 6.97-11.55) was 3.7-times greater than the higher handgrips strength group (mean, 2.47 days/years; 95\% CI, 0.64-4.31). Therefore, our findings suggest a decreased handgrip strength was a predictor on the increased risk of hospitalization and medical use. Moreover, these observations remain consistent when after adjusting potential confounding factors.

Although several previous studies have shown associations of physical fitness with hospitalization in older adults [20], this 
study is the first study to examine the prospective association of handgrip strength with the risk of hospitalization and medical use in Korean population. Compared with some crosssectional studies, we showed comparable beneficial medical health results for higher handgrip strength. Furthermore, our findings support the emerging cross-sectional population studies suggesting that higher handgrip strength is associated with lower risk of chronic diseases, when compared to lower handgrip strength.

The muscular strength is known to have a role in various metabolic responses. Thus, lower muscular strength could have a harmful chronic diseases effect via endocrine and metabolic mechanisms [21]. For example, skeletal muscle is responsible for $75 \%$ of post prandial glucose uptake and contributes to glucose homeostasis [22]. Moreover, age related muscle decline induces a $2-3 \%$ decline in basal metabolic rate (BMR) per decade after age 20 years and $4 \%$ per decade after age 50 years, resulting from concomitant loss of mitochondrial volume density and oxidative capacity [23]. In fact, several previous studies report that loss of skeletal muscle mass and strength is related to metabolic syndrome, which induces risk factors for type 2 diabetes and CVD [24]. We previously investigated an association of sarcopenia based on handgrip strength and the risk of cognitive impairment using a large population database [25], and other studies showed the relationship sarcopenia with musculoskeletal disorders such as mobility impairment, physical disability, falls, fractures, and osteoporosis [21,26-29].

There are several factors that may explain the effect of lower handgrip strength on the risk of hospitalization and medical use. It is intuitive to suggest that having low muscle mass and strength may lead to more functional limitation and more metabolic diseases, which may be caused by geriatric conditions [30,31]. In fact, loss of skeletal muscle mass have been reported to be related with increased geriatric conditions i.e. falls, delirium, malnutrition, and disability, that also contributes to chronic diseases [31].

\section{Strengths and limitations}

Our study has several strengths and limitations. We measured decreased muscular strength by measuring the handgrip strength among middle and older aged of large general population. Moreover, we controlled important potential confounding factors that might influence muscular strength and medical use including demographics, lifestyle, and clinical health status. Also, we could infer cause-and-effect association between handgrip strength and medical health care because of longitudinal study design.

In contrast, several limitations should be considered. We could not evaluate muscle mass. We only evaluated handgrip strength as muscular function. Skeletal muscle mass as well as muscular function is significant for quality of life and prevention of disease in the middle-aged and elderly population. The Asian and European Working Group on Sarcopenia in Older People recommend the definition of sarcopenia to consider both skeletal muscle mass and muscular function including handgrip strength. Additional studies involving muscle mass may be needed.

\section{Conclusions}

Our findings suggest that lower handgrip strength was associated with increased the risk of hospitalization and use of medical care in Korean population. This study highlights the maintaining of muscular strength may play an important role in the reduction of risk for hospitalization and medical use by preventing chronic diseases.

\section{Acknowledgments}

This work was supported by the Korea Maritime \& Ocean University Research Fund (2018-0290-01).

\section{Conflict of interests}

The authors declare no conflict interests.

\section{References}

1. Goodpaster BH, Park SW, Harris TB, et al. The loss of skeletal muscle strength, mass, and quality in older adults: the health, aging and body composition study. J Gerontol A Biol Sci Med Sci. 2006; 61(10):1059-1064. 
2. Mitchell WK, Williams J, Atherton P, Larvin M, Lund J, Narici M. Sarcopenia, dynapenia, and the impact of advancing age on human skeletal muscle size and strength; a quantitative review. Front Physiol. 2012; 3:260.

3. Woodrow G. Body composition analysis techniques in the aged adult: indications and limitations. Curr Opin Clin Nutr Metab Care. 2009; 12(1):8-14.

4. Gallagher D, Visser M, De Meersman RE, et al. Appendicular skeletal muscle mass: effects of age, gender, and ethnicity. J Appl Physiol (1985). 1997; 83(1):229-239.

5. Kim JH CJ, Park YS. Relationship between sarcopenic obesity and cardiovascular disease risk as estimated by the Framingham risk score. J Korean Med Sci 2015; 30: 264-271.

6. Yoon J, Kim J, Choi Y-H. Associations of Sarcopenia with Rheumatoid Arthritis and Osteoarthritis in Korean Adults: the Korea National Health and Nutrition Examination Survey 2008-2011. J Kinesiol. 2017; 19(3):61 - 70 .

7. An KO, Kim J. Association of sarcopenia and obesity with multimorbidity in Korean adults: a nationwide crosssectional study. J Am Med Dir Assoc. 2016; 17(10):960. e961-960. e967.

8. Janssen I, Heymsfield S B, Ross R. Low relative skeletal muscle mass (sarcopenia) in older persons is associated with functional impairment and physical disability. $J$ Am Geriatr Soc. 2002; 50(5):889-896.

9. Biolo G, Cederholm T, Muscaritoli M. Muscle contractile and metabolic dysfunction is a common feature of sarcopenia of aging and chronic diseases: from sarcopenic obesity to cachexia. Clinical Nutrition. 2014; 33(5):737-748.

10. Jeong S, Kim J. Prospective association of handgrip strength with risk of new-onset cognitive dysfunction in Korean adults: a 6-year national cohort study. Tohoku J Exp Med. 2018; 244(2):83-91.

11. Lee S, Kim TN, Kim SH. Sarcopenic obesity is more closely associated with knee osteoarthritis than is nonsarcopenic obesity: a cross-sectional study. Arthritis
Rheum. 2012; 64(12):3947-3954.

12. Lim S, Kim JH, Yoon JW, et al. Sarcopenic obesity: prevalence and association with metabolic syndrome in the Korean Longitudinal Study on Health and Aging (KLoSHA). Diabetes Care. 2010; 33(7):1652-1654.

13. Stenholm S, Alley D, Bandinelli S, et al. The effect of obesity combined with low muscle strength on decline in mobility in older persons: results from the InCHIANTI study. Int J Obes (Lond). 2009; 33(6):635-644.

14. Ahmadi B, Alimohammadian $M$, Yaseri $M$, et al. Multimorbidity: Epidemiology and Risk Factors in the Golestan Cohort Study, Iran: A Cross-Sectional Analysis. Medicine (Baltimore). 2016; 95(7):e2756.

15. Li D, Guo G, Xia L, et al. Relative Handgrip Strength Is Inversely Associated with Metabolic Profile and Metabolic Disease in the General Population in China. Front Physiol. 2018; 9:59.

16. An K, Kim J. Associations between Handgrip Strength with Adverse Cardiometabolic Health among Representative Korean Adults. Int J Environ Res Public Health. 2020; 17:269.

17. The IPAQ group. Guidelines for Data Processing and Analysis of the International Physical Activity Questionnaire (IPAQ) -Short and Long Forms. 2005; http://www.ipaq.ki.se/scoring.pdf. (Accessed 05 Nov, 2016)

18. Waxman A. WHO global strategy on diet, physical activity and health. Food Nutr Bull. 2004; 25(3):292-302.

19. World Health Organization. World Health Organization (WHO) Global Strategy on Diet, Physical Activity and Health. 2004; http://www.who.int/dietphysicalactivity/ strategy/eb11344/strategy_english_web.pdf?ua=1. (Accessed 11 Feb, 2015)

20. Hida T, Shimokata H, Sakai Y, et al. Sarcopenia and sarcopenic leg as potential risk factors for acute osteoporotic vertebral fracture among older women. Eur Spine J. 2015.

21. Cleasby ME, Jamieson P, Atherton PJ. Insulin resistance and sarcopenia: mechanistic links between common co-morbidities. J Endocrinol. 2016.

22. DeFronzo RA, Tripathy D. Skeletal muscle insulin 
resistance is the primary defect in type 2 diabetes. Diabetes Care. 2009; 32 Suppl 2:S157-163.

23. Conley KE, Jubrias SA, Esselman PC. Oxidative capacity and ageing in human muscle. J Physiol. 2000; $526 \mathrm{Pt}$ 1:203-210.

24. Kim TN, Yang SJ, Yoo HJ, et al. Prevalence of sarcopenia and sarcopenic obesity in Korean adults: the Korean sarcopenic obesity study. Int J Obes (Lond). 2009; 33(8):885-892.

25. Jang JY, Kim J. Association between handgrip strength and cognitive impairment in elderly Koreans: a populationbased cross-sectional study. J Phys Ther Sci. 2015; 27(12):3911-3915.

26. Pereira FB, Leite AF, Paula AP. Relationship between presarcopenia, sarcopenia and bone mineral density in elderly men. Arch Endocrinol Metab. 2015; 59(1):59-65.

27. Janssen I, Baumgartner RN, Ross R, Rosenberg IH, Roubenoff R. Skeletal muscle cutpoints associated with elevated physical disability risk in older men and women. Am J Epidemiol. 2004; 159(4):413-421.
28. Bouchard DR, Dionne IJ, Brochu M. Sarcopenic/obesity and physical capacity in older men and women: data from the Nutrition as a Determinant of Successful Aging (NuAge)-the Quebec longitudinal Study. Obesity (Silver Spring). 2009; 17(11):2082-2088.

29. Janssen I, Heymsfield SB, Ross R. Low relative skeletal muscle mass (sarcopenia) in older persons is associated with functional impairment and physical disability. $J$ Am Geriatr Soc. 2002; 50(5):889-896.

30. Van Ancum JM, Scheerman K, Pierik VD, et al. Muscle Strength and Muscle Mass in Older Patients during Hospitalization: The EMPOWER Study. Gerontology. 2017; 63(6):507-514.

31. Van Ancum JM, Scheerman K, Jonkman NH, et al. Change in muscle strength and muscle mass in older hospitalized patients: A systematic review and metaanalysis. Exp Gerontol. 2017; 92:34-41. 